\title{
Nuclear metallothionein in oral squamous cell carcinoma: clinicopathological parameters and patient survival
}

Girlene Maria do Rego BEZERRA(a) Flávia Sirotheau Corrêa PONTES(a) Nicolau CONTE NETO(a) Liliane Silva do NASCIMENTO(a) Lucas Lacerda de SOUZA ${ }^{(a)}$ Décio dos Santos PINTO JUNIOR(b) Hélder Antônio Rebelo PONTES ${ }^{(a)}$

(a) João de Barros Barreto University Hospital, Belém, PA, Brazil.

(b) UniversidAde de São Paulo - USP, School of Dentistry, Department of Stomatology, São Paulo, SP, Brazil.

Declaration of Interest: The authors certify that they have no commercial or associative interest that represents a conflict of interest in connection with the manuscript.

Corresponding Author:

Lucas Lacerda de Souza

E-mail: lucaslac@hotmail.com

https://doi.org/10.1590/1807-3107bor-2018.vol32.0105

Submitted: October 11, 2017

Accepted for publication: April 04, 2018

Last revision: August 29, 2018
Abstract: The aim of this study was to identify the immunoexpression of methallothionein in oral squamous cell carcinoma as well as to address the correlation with clinical features, histological grade and patient survival. Samples were collected from 93 patients with tongue squamous cell carcinoma who presented for follow-up. Immunohistochemical expression of methallothionein in all groups was performed. The scoring system has previously been published by Tsurutani in 2005, which is based on intensity and distribution of staining. We used Kappa index to evaluate the degree of observers' agreement under metallothionein immunostaining and histological grade. Associations between methallothionein expression and clinical parameters (age, gender, smoking, tumor size, lymph node metastasis and disease stage) were examined for statistical significance using the chi-squared test. The overall survival rates were estimated by the Kaplan-Meier method and the relationship between protein expression and survival was compared using the log-rank test $(\mathrm{p}<0.05)$. Our results showed no statistically significant association between methallothionein immunostaining and the selected clinicopathological variables. Immunohistochemistry results showed positive nuclear immunostaining for metallothionein in $62,37 \%$ (58/93) and negative for metallothionein 37,63\% (35/93). The degree of examiners agreement by Kappa varied from substantial to perfect and both metallothionein immunostaining and histological grade were explored. The present study suggests that positive methallothionein expression found in tongue squamous cell carcinoma may not help to predict survival in the analyzed samples, as well as no relation between the protein and histological grade and clinical features was observed. In conclusion, the present study suggests that metallothionein is not associated with tongue squamous cell carcinoma clinicopathological characteristics and aggressiveness.

Keywords: Mouth Neoplasms; Survival; Immunohistochemistry.

\section{Introduction}

Oral squamous cell carcinoma (OSCC) is the most common form of oral cancer, representing more than $90 \%$ of all malignant neoplasms in this location. ${ }^{1}$ Despite the recent advances in surgery and improvements in radio and chemotherapy, OSCC continues to pose a significant therapeutic problem. . $3,34^{-}$ 
Metallothioneins (MT) comprise a group of low molecular weight $(6-7 \mathrm{kDa})$, cysteine-rich, intracellular proteins with high affinity for heavy metal ions. ${ }^{5}$ This protein is known to act in detoxification of heavy metals, homeostasis of essential metals, antioxidation against reactive oxygen species, protect against DNA damage and oxidative stress as well as in cell survival, angiogenesis, apoptosis and increase proliferation. ${ }^{6}$ In addition, its overexpression seems to be related to resistance to radiation and chemotherapeutic agents. ${ }^{78}$ Thus, MT has been studied as a prognostic factor for different human cancers. ${ }^{9,10,11}$ We showed recently a significant increase in positive immunohistochemical staining for MT-1/2 proteins when normal oral mucosa was compared to severe dysplasia and to OSCC as well as a positive correlation with severity of dysplasia of oral leukoplakia, with the lowest MT-1/2 expression found in mild dysplastic lesions and the highest in severe dysplasia. ${ }^{12}$

The transcriptional control of MT induction and changes in its nuclear/cytoplasmic localization during cell proliferation and differentiation suggest that altered levels of nuclear MT can be expected in any situation where there is abnormal cell growth, such as cancer. ${ }^{9}$ Moreover, cases characterized by a positive nuclear MT-1/2 immunostaining yielded higher p53 expression levels. MT molecules were found to be able to remove zinc ions from p53 protein, what results in its inactivity. Inactivation of p53 protein in neoplastic cells results in their excessive proliferation and inhibition of apoptotic processes. ${ }^{10,13}$

A better understanding of the cellular molecules involved in the mechanisms of OSCC pathogenesis is important to identify prognostic markers that reflect disease aggressiveness, to provide more effective forms of therapy and may improve patient survival. In this context, the aim of this study was to examine by immunohistochemistry in samples of OSCC the correlation of MT nuclear expression with selected clinical and pathological variables of the disease as well as with the patient survival. As studies about the expression of MT in OSCC confined to nuclear compartment are limited, we conducted the present study to obtain more accurate information about the clinical importance of nuclear MT expression in OSCC.

\section{Methodology}

\section{Specimens and inclusion criteria}

A total of 93 paraffin-embedded biopsy specimens of tongue squamous cell carcinoma from 67 (72,5\%) males and $26(27,5 \%)$ females with a mean age of 60 years (range, 27-85) were selected in the period between January 1999 and December 2014 from the Service of Oral Pathology of the João de Barros Barreto University Hospital (Pará, Brazil) to evaluate the immunohistochemical expression of the metallothionein protein and to correlate with survival. The patients were monitored for up to 62 months after diagnose. Samples were selected from patients (with a diagnosis confirmed by histopathology) who had primary tumours of the oral cavity with surgery as the only treatment modality. The smokers consumed at least 10 cigarettes per day. The mean follow-up of the patients was 28.02 months (range, 0-62). A total of 15 patients with a change in staging in the period between diagnosis and surgery were excluded. In our study, there were no cases with distant metastasis. The required data were obtained from patient records, summarised on standardized forms and stored in a database. A total of 93 paraffin-embedded biopsy specimens of OSCC were subjected to evaluation of histological grade and clinicopathological features. The primary tumor was clinically staged according to the TNM classification defined by the 2009 International Union Against Cancer (UICC) ${ }^{14}$ and the 2010 American Joint Committee on Cancer (AJCC).$^{15}$ The histological grade assessment followed the parameters of the World Health Organization ${ }^{16}$ and was carried out by two pathologists without prior knowledge of the clinical data of the patients. The relationships between metallothionein protein and clinicopathological features, size of tumour (T), infiltration of metastatic lymph nodes $(\mathrm{N})$, stage, smoking and the histological grade, were assessed. The ethical committee of the João de Barros Barreto University Hospital approved this work under approval number 51641/12.

\section{Immunohistochemistry}

Sections were dewaxed with xylene and hydrated in an ethanol series. For antigen retrieval, sections that received antimetallothionein antibody were immersed 
in $10 \mathrm{mM}$ monohydrated citrate buffer solution ( $\mathrm{pH}$ 6.0) and heated in a microwave oven at $95^{\circ} \mathrm{C}$ for $15 \mathrm{~min}$. Peroxidase activity was blocked with $6 \%$ hydrogen peroxide and methanol solution in two baths for $15 \mathrm{~min}$ each at room temperature. After washing with Tris buffer ( $\mathrm{pH}$ 7.4), the slides were incubated with the primary antibody anti -metallothionein isoforms 1 and 2 (DAKO Corporation, Glostrup, Denmark) E9 clone, dilution $1 / 800$, incubated for $60 \mathrm{~min}$. The sections were subsequently exposed to avidin-biotin complex (LSAB-Kit + HRP; DakoCytomation, Carpinteria, USA) and to 3, 30-diaminobenzidine chromogen (DAB+; DakoCytomation). The sections were counterstained with Meyer's haematoxylin, dehydrated in ethanol, cleared in xylene and mounted. Slices of breast adenocarcinoma were used as the positive control, and the negative control was obtained by omitting the primary specific antibody during the reaction. Immunostained sections were examined by light microscopy at 409 magnification, and digital images were captured using an electron microscope model Eclipse Nikon Ci-POL (Nikon Metrology Europe NV, Leuven, Belgium). All sections were evaluated without knowledge of clinical status by two pathologists, under a fixed focus. The staining was considered positive when cells with brown staining were observed in the nucleus compartment, indicating the presence of DAB in the immunohistochemistry reaction.

The scoring system has previously been published in the literature. ${ }^{18}$ The analysis was based on intensity and distribution of staining. The distribution of stained cells was analyzed as follows: $0(0 \%), 1$ (1-50\%) and 2 (51-100\%). The intensity of staining was rated as follows: 0 (no staining), 1 (mild staining), 2 (moderate staining) and 3 (strong staining). The staining pattern of the specimens was defined by the sum of the values found in the distribution of data for the intensity of immunostaining, thus obtaining the final record (FR) as follows: FR0, FR2, FR3, FR4 and FR5. Using this method, FR0 and FR2 were considered negative staining, while FR3, FR4 and FR5 were considered positive staining. Two independent pathologists blinded to the experimental groups evaluated the immunostained sections. In the event of a disagreement, the two pathologists conferred to achieve a consensus. A record card was used to register the inter-observer agreement among the variables through the Kappa test statistics to compare the agreement among individual pathologists around metallothionein immunostaining and histological grade.

\section{Statistical analysis}

The data was analysed using the Statistical Package for Social Sciences software for Windows, version 18.0 (SPSS Inc, Chicago, IL, USA). Associations between metallothionein expression and clinicopathological parameters were examined for statistical significance using a chi-square test. Overall survival rates were estimated by the Kaplan-Meier method and compared using a log rank test. A p-value of $<0.05$ was considered significant.

\section{Results}

\section{Clinical profiles of patients with OSCC and evaluation of histological grade}

A total of 93 samples were included in the analysis. Patient characteristics are summarised in Table. Moderately differentiated tumours (48,38\%), followed by poorly differentiated $(35.48 \%$ ) tumours, were the most represented in our sample. Well-differentiated tumours represented only $15.05 \%$. The chi-square test showed no significant difference for the clinical and histological profiles analyzed.

\section{Metallothionein immunostaining}

Immunohistochemistry results showed positive nuclear immunostaining for metallothionein in $62,37 \%$ (58/93) samples and negative immunostaining for metallothionein in 37,63\% (35/93) samples. The immunostaining was observed in cells located at the periphery and at the center of the tumours islands; however, wherever keratin pearls were present, MT immunostaining was restricted to the basal and parabasal cells (Figure 1). In order to compare the agreement among individual pathologists the unweighted kappa statistics were calculated which were found to be significant $(p<0.05)$, evidencing results varying from substantial to perfect when metallothionein immunostaining and histological grade were explored. 
Table. Association between metallothionein immunostaining and clinicopathological variables.

\begin{tabular}{|c|c|c|c|}
\hline \multirow{2}{*}{$\begin{array}{l}\text { Clinicopathological } \\
\text { features } \\
n=93\end{array}$} & \multicolumn{3}{|c|}{$\begin{array}{l}\text { Metallothionein nuclear } \\
\text { immunostaining }\end{array}$} \\
\hline & Negative & Positive & $p$-value \\
\hline Age & & & 0.74 \\
\hline$\leq 40$ year & 4 & 4 & \\
\hline$>40$ years & 31 & 54 & \\
\hline Gender & & & 1.0 \\
\hline Female & 18 & 29 & \\
\hline Male & 17 & 29 & \\
\hline Alcohol intake & & & 0.61 \\
\hline No & 15 & 28 & \\
\hline Yes & 20 & 30 & \\
\hline Smoking & & & 0.61 \\
\hline No & 23 & 41 & \\
\hline Yes & 12 & 17 & \\
\hline Size tumour $(T)$ & & & 0.76 \\
\hline 1 or 2 & 15 & 23 & \\
\hline 3 or 4 & 20 & 35 & \\
\hline Lymph node metastasis (N) & & & 0.74 \\
\hline 0 or 1 & 27 & 43 & \\
\hline 2 or 3 & 8 & 15 & \\
\hline Stage & & & 0.94 \\
\hline | or || & 13 & 22 & \\
\hline III or IV & 22 & 36 & \\
\hline Histological grade & & & 0.93 \\
\hline Well differentiated & 5 & 10 & \\
\hline Moderate & 18 & 27 & \\
\hline Poorly differentiated & 12 & 21 & \\
\hline Overall survival & & & 0.08 \\
\hline Alive & 3 & 40 & \\
\hline Dead & 4 & 46 & \\
\hline
\end{tabular}

\section{Overall survival}

Overall survival was defined as the period between the date of diagnosis of the disease until the last follow-up or death (Table). Log rank test showed that there was not a statistically significant association between survival rate and metallothionein immunostaining ( $p=0.0835$ ) (Figure 2). The Kaplan-Meier curve showed that the probability of survival for a patient after 1 month of monitoring the disease was $96.5 \%$ and that the passage of time tended to reduce this probability, as shown in Figure 3. The probability of a patient surviving after 60 months of follow-up was $24.83 \%$.

\section{Discussion}

Metallothionein has been implicated in several aspects of cancer pathobiology, such as differentiation, proliferation, apoptosis and invasion. ${ }^{18}$ Moreover, the carcinogen from tobacco smoking benzo[a]-pyrene $(\mathrm{BaP})$, a polycyclic aromatic hydrocarbon, also triggers MT-1 overexpression..$^{19}$ We showed recently a significant increase in positive immunohistochemical staining for MT- $1 / 2$ protein when normal oral mucosa was compared to severe dysplasia and to OSCC as well as a positive correlation with severity of dysplasia of oral leukoplakia, with the lowest MT-1/2 expression found in mild dysplastic lesions and the highest in severe dysplasia, ${ }^{12}$ then the present study aimed to assess MT nuclear expression immunohistochemically in oral SCC specimens and to evaluate clinicopathological parameters, including overall survival time.
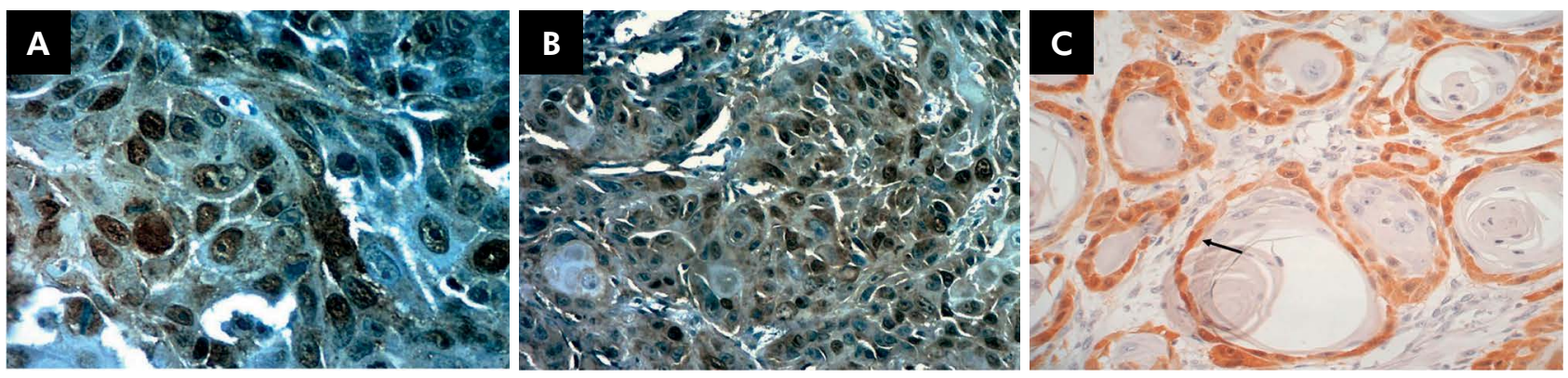

Figure 1. Immunoexpression of metallothionein protein in OSCC epithelium, nuclear immunostaining (x400 original magnification) (a). The immunostaining was located at the periphery and at the center of the tumor island (b). The immunostaining around the pearls of keratin (c). 


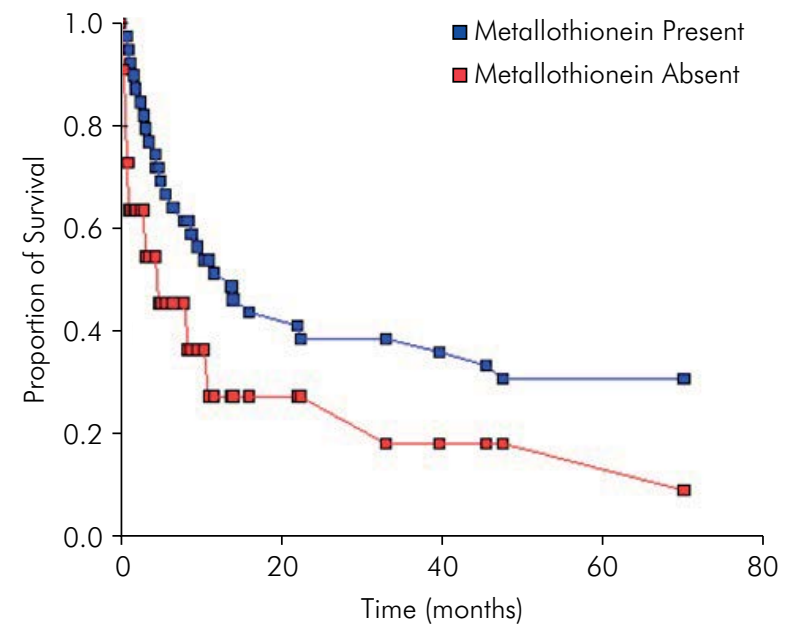

Figure 2. Survival rate association with metallothionein immunostaining ( $p=0.0835)$.

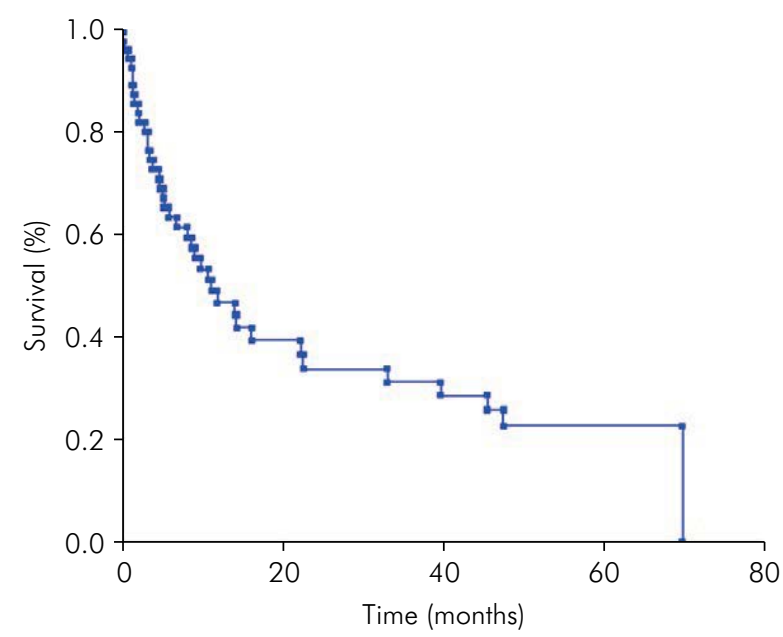

Figure 3. Kaplan-Meier graphic with overall survival curve.

There is no conclusive data on the functional significance of MT distribution in different cellular compartments. In mitotically inactive cells (G0 phase), the expression of MT can be detected in the cytoplasm while in dividing cells its activity becomes shifted to the nucleus. The highest cytoplasmic expression of MT is observed at the end of G1 phase and at the G1/S threshold while the peak of accumulation of MT in the nucleus can be detected in phases $S$ and G2. The translocation of MT into the nucleus during G1/S phase in tumor cells suggests that MT facilitates cell proliferation by donating zinc ions to various transcription factors. ${ }^{9,20,21,22}$ In this sense, it is of high importance to distinguish between MT-I+II expression both in subcellular compartments (e.g. cytoplasmic vs. nuclear).

MT could exert its anti-apoptotic effects in oral squamous cell carcinomas by blocking the action of $\mathrm{p} 53$ protein. Douglas-Jones et al. ${ }^{23}$ suggested that MT could remove Zn of p53 by blocking the activity of inducing programmed cell death. Ostrakhovitch et al. ${ }^{24}$ showed, in breast cancer cell culture, the interaction between MT1 and p53, suggesting that co-expression of MT1 and p53 may be involved in the regulation of apoptosis in these cells. In addition, it has been shown that combined Zn and p53 proteins deficiency allows extreme sensitivity to malignant transformation, using $4 \mathrm{NQO}$ (4-nitroquinoline 1-oxide) at low concentrations at an experimental model of oral carcinogenesis conducted in mice. ${ }^{25}$ Finally, cases characterized by a positive nuclear MT-1/2 immunostaining, yielded higher p53 expression levels. ${ }^{13}$ This underlies the earlier observed role of MT- $1 / 2$ in regulation of p53 expression by influencing zinc ion cell homeostasis.

Despite the immunostaining observed in this study being sometimes restricted to the nucleus, sometimes restricted to the cytoplasm and sometimes found in both compartments, in view of the above considerations, the present study focused only in specimens with positive nuclear staining or nuclear and cytoplasmic staining simultaneously. To our knowledge, the present study is the only investigation in which the intensity and distribution of MT nuclear expression in OSCC is analyzed together and related with clinicopathological characteristics.

A mosaic staining pattern for the MT protein was observed in the squamous cell carcinomas investigated in our study, with strongly immunostained cells in some areas, while other areas, on the same blade, showed negative results. We believe this mosaic pattern may be the result of cell phenotypic differences acquired during neoplastic progression.

MT immunoreactivity was frequently detected in the peripheral cell layers around keratins pearls, where the tumour cells were less differentiated, whereas the cell layers inside the keratin pearl, 
where the tumour cells were more differentiated and keratinized, showed weak or negative MT immunoreactivity (Figure 1C). For Sundelin et al., ${ }^{26}$ peripheral staining of MT reproduces the staining pattern found in normal epithelium and can help divert these cells from programmed cell death. The pattern of immunostaining observed in the present study was similar to the one found by Cardoso et al. ${ }^{11}$ and Theocharis et al. ${ }^{18}$

The tongue carcinoma has been shown to have high local failure and poor survival rates compared with other anatomical sites in the oral cavity. ${ }^{27}$ In this context, the present study focused on anatomical site to assess overall survival time. Analysis of the MT protein expression did not promote statistically significant results when associated with survival of patients with squamous cell carcinoma involved in this study, similar results to those found by Szelachowska et al., ${ }^{28}$ where MT nuclear staining was associated with overall survival time. In another study of Cardoso et al., no influence on patients survival was observed, when MT-1/2 expression was analyzed alone. Interestingly, a combined analysis of this protein with p53 expression, showed that high expression of both these markers predicted poor outcome of patients with OSCC..$^{13}$

It is worth mentioning that the study by Theocharis et al. ${ }^{18}$ found a statistically significant relationship when evaluating the intensity of MT staining with overall survival time. Their study evaluated the staining intensity separately of the staining distribution, finding no statistically significant results when the relation of MT immunostaining with the staining distribution were evaluated singly. In our study, we evaluated the intensity and the distribution of staining together. Therefore, there is no methodological parameters for comparison between the two studies.

No significant statistical correlation was observed among the clinicopathological parameters examined and MT imunostaining in malignant cells. Similar results to those found by Theocharis et al. ${ }^{18} 2011$ which separately assessed MT distribution and staining intensity. Some studies in the literature did not show correlations with primary tumour size and grade of tumour differentiation. ${ }^{11,13,19,28,29,30}$ However studies of
Lee etal., ${ }^{19}$ Szelachowska etal. ${ }^{28}$ and Szelachowska et al. ${ }^{30}$ showed a positive correlation between lymph node involvement and the intensity of MT expression. Szelachowska et al..$^{28}$ analyzed cytoplasmatic and nuclear MT-1/2 expressions separately.

Zavras et al. ${ }^{31}$ demonstrated that certain MT-1 allotypes may be genetic risk factors which increase susceptibility to OSCC and were consistently associated with a higher risk for advanced stage, increased involvement of lymph node and dedifferentiation of the tumor.

The significance of subcellular localization of MT at the nucleus/cytoplasm in OSCC is also not well understood. In our study, we also compared the exclusive cytoplasmic localization of MT with the clinical/histological variables (data not shown), but no statistically significant results of MT expression with any of the investigated characteristics were found, which means that, in our sample, the sublocation of MT was unrelated to the aggressiveness of the OSCC.

The differences between our study and the others which developed associations between histological grade, clinical features and positive prognostic with MT may be related to the number of analyzed samples and the heterogeneity of the samples.

In conclusion, the present study showed that whilst MT is highly expressed in human OSCC, it is not correlated with clinicopathological characteristics. Therefore, this protein does not seem to exert a crucial role in the aggressiveness of this tumor in the cases evaluated. The accumulated knowledge of MT expression in relation to clinicopathological parameters has provided contradictory results, possibly due to the methodology of evaluation; the specificity of OSCC affected sites used in the studies, or because of tumor-specific characteristics. Further studies are necessary to understand the participation of MT in oral carcinogenesis.

\section{Acknowledgments}

The authors acknowledge the Pará State Research Foundation (FAPESPA) for financial support and scholarship grants. 


\section{References}

1. Anaya-Saavedra G1, Ramírez-Amador V, Irigoyen-Camacho ME, Zimbrón-Romero A, Zepeda-Zepeda MA. Oral and pharyngeal cancer mortality rates in México, 1979-2003. J Oral Pathol Med. 2008 Jan;37(1):11-7.

2. Chen JS, Pardo FS, Wang-Rodriguez J, Chu TS, Lopez JP, Aguilera $\mathrm{J}$ et al. EGFR regulates the side population in head and neck squamous cell carcinoma. Laryngoscope. 2006 Mar;116(3):4016. https://doi.org/10.1097/01.mlg.0000195075.14093.fb

3. Chin D, Boyle GM, Williams RM, Ferguson K, Pandeya N, Pedley J, et al. Novel markers for poor prognosis in head and neck cancer. Int J Cancer. 2005 Feb;113(5):789-97. https://doi.org/10.1002/iic.20608

4. Vora HH, Shah NG, Patel DD, Trivedi TI, Chikhlikar PR. Prognostic significance of biomarkers in squamous cell carcinoma of the tongue: multivariate analysis. J Oncol. 2003 Jan;82(1):34-50. https://doi.org/10.1002/jso.10183

5. Thirumoorthy N, Shyam Sunder A, Manisenthil Kumar K, Senthil Kumar M, Ganesh G, Chatteriee M. A review of metallothionein isoforms and their role in pathophysiology. World J Surg Oncol. 2011 May;9(1):54. https://doi.org/10.1186/1477-7819-9-54

6. Higashimoto $M$, Isoyama N, Ishibashi S, Inove M, Takiguchi $M$, Suzuki $S$ et al. Tissue-dependent preventive effect of metallothionein against DNA damage in dyslipidemic mice under repeated stresses of fasting or restraint. Life Sci. 2009;84(1718):569-75. https://doi.org/10.1016/j.lfs.2009.01.022

7. Gallicchio LM, Flaws JA, Fowler BA, loffe OB. Metallothionein expression in invasive and in situ breast carcinomas. Cancer Detect Prev. 2005;29(4):332-7. https://doi.org/10.1016/i.cdp.2005.06.011

8. Satoh M, Cherian MG, Imura N, Shimizu H. Modulation of resistance to anticancer drugs by inhibition of metallothionein synthesis. Cancer Res. 1994 Oct;54(20):5255-7.

9. Cherian MG, Jayasurya A, Bay BH. Metallothioneins in human tumors and potential roles in carcinogenesis. Mutat Res. 2003 Dec;533(1-2):201-9. https://doi.org/10.1016/j.mrfmmm.2003.07.013

10. Pastuszewski W, Dziegiel P, Krecicki T, Podhorska-Okolow M, Ciesielska U, Gorzynska E et al. Prognostic significance of metallothionein, p53 protein and $\mathrm{Ki}-67$ antigen expression in laryngeal cancer. Anticancer Res. 2007 Jan-Feb;27(1A):335-42.

11. Cardoso SV, Barbosa HM, Candellori IM, Loyola AM, Aguiar MF. Prognostic impact of metallothionein on oral squamous cell carcinoma. Virchows Arch. 2002;441(2):174-8. https://doi.org/10.1007/s00428-001-0588-3

12. Pontes HA, Xavier FCA, Silva TS, Fonseca FP, Paiva HB, Pontes FS et al. Metallothionein and $\mathrm{p}$-Akt proteins in oral dysplasia and in oral squamous cell carcinoma: an immunohistochemical study. J Oral Pathol Med. 2009 Sep;38(8):644-50. https://doi.org/10.1111/j.1600-0714.2009.00787.x
13. Cardoso SV, Silveira-Júnior JB, Machado VC, De-Paula AM, Loyola AM, Aguiar MC. Expression of metallothionein and p53 antigens are correlated in oral squamous cell carcinoma. Anticancer Res. 2009 Apr;29(4):1189-93.

14. Sobin LH, Wittekind CH. TNM classification of malignant tumors. 7. ed. New York: Springer; 2009.

15. Edge SB, Compton CC. The American Joint Committee on Cancer: the 7th edition of the AJCC cancer staging manual and the future of TNM. Ann Surg Oncol 2010 Jun;17(6):14714. https://doi.org/10.1245/s10434-010-0985-4

16. Müller S. Update from the 4th Edition of the World Health Organization of Head and neck tumours: tumours of the oral cavity and mobile tongue. Head Neck Pathol. 2017 Mar;11(1):33-40. https://doi.org/10.1007/s12105-017-0792-3

17. Tsurutani J, Castillo SS, Brognard J, Granville CA, Zhang C, Gills JJ et al. Tobacco components stimulate Akt-dependent proliferation and NFkappaB-dependent survival in lung cancer cells. Carcinogenesis. 2005 Jul;26(7):1182-95. https://doi.org/10.1093/carcin/bgi072

18. Theocharis S, Klijanienko J, Giaginis C, Rodriguez J, Jouffroy T, Girod A et al. Metallothionein expression in mobile tongue squamous cell carcinoma: associations with clinicopathological parameters and patient survival. Histopathology. 2011 Sep;59(3):514-25. https://doi.org/10.1111/j.1365-2559.2011.03947.x

19. Lee SS, Yang SF, Tsai CH, Chou MC, Chou MY, Chang YC The upregulation of metallothionein-1 expression in areca quid chewing-associated oral squamous cell carcinoma. Oral Oncol. 2008;44:180-6. https://doi.org/10.1016/j.oraloncology.2007.01.019

20. Abdel-Mageed AB, Agrawal KC. Activation of nuclear factor kappaB: potential role in metallothionein-mediated mitogenic response. Cancer Res. 1998 Jun;58(11):2335-8.

21. Eckschlager T, Adam V, Hrabeta J, Figova K, Kizek R. Metallothioneins and cancer. Curr Protein Pept Sci. 2009 Aug;10(4):360-75. https://doi.org/10.2174/138920309788922243

22. Piotr D, Bartosz P, Marzena PO. Expression of metallothionein in oral cancer. In Ogbuereke KUE. Oral cancer. Rijeka: Itech; 2012 [cited 2012 March 14]. Available from: http:// www.intechopen.com/books/oral-cancer/expression-ofmetallothionein-in-oral-cancer

23. Douglas-Jones AG, Navabi H, Morgan JM, Jasani B. Immunoreactive p53 and metallothionein expression in duct carcinoma in situ of the breast. No correlation. Virchows Arch. 1997 May;430(5):373-9. https://doi.org/10.1007/s004280050046

24. Ostrakhovitch EA, Olsson PE, Jiang S, Cherian MG. Interaction of metallothionein with tumor supressor p53 protein. FEBS Lett. 2006;580(5):1235-8. https://doi.org/10.1016/i.febslet.2006.01.036 
- Nuclear metallothionein in oral squamous cell carcinoma: clinicopathological parameters and patient survival

25. Fong LY, Jiang Y, Farber JL. Zinc deficiency potentiates induction and progression of lingual and esophageal tumors in p53-deficient mice. Carcinogenesis. 2006 Jul;27(7):1489-96. https://doi.org/10.1093/carcin/bgl012

26. Sundelin K, Jadner M, Norberg-Spaak L, Davidsson A, Hellquist HB. Metallothionein and Fas (CD95) are expressed in squamous cell carcinoma of the tongue. Eur J Cancer. 1997 Oct;33(11):1860-4. https://doi.org/10.1016/S0959-8049(97)00216-5

27. Shiboski CH, Schmidt BL, Jordan RC. Tongue and tonsil carcinoma: increasing trends in the U.S. population ages 20-44 years. Cancer. 2005 May;103(9):1843-9. https://doi.org/10.1002/cncr.20998

28. Szelachowska J, Dziegiel P, Jelen-Krzeszewska J, Jelen M, Tarkowski R, Wlodarska I et al. Prognostic significance of nuclear and cytoplasmic expression of metallothioneins as related to proliferative activity in squamous cell carcinomas of oral cavity. Histol Histopathol. 2008 Jul;23(7):843-51. https://doi.org/10.14670/HH-23.843

29. Muramatsu Y, Hasegawa Y, Fukano H, Ogawa T, Namuba $M$, Mouri $\mathrm{K}$ et al. Metallothionein immunoreactivity in head and neck carcinomas; special reference to clinical behaviors and chemotherapy responses. Anticancer Res. 2000 JanFeb;20(1A):257-64.

30. Szelachowska J, Dziegiel P, Jelen-Krzeszewska J, Jelen M, Tarkowski R, Spytkowska B et al. Correlation of metallothionein expression with clinical progression of cancer in the oral cavity. Anticancer Res. 2009 Feb;29(2):589-95.

31. Zavras Al, Yoon AJ, Chen MK, Lin CW, Yang SF. Metallothionein-1 genotypes in the risk of oral squamous cell carcinoma. Ann Surg Oncol. 2011 May;18(5):1478-83. https://doi.org/10.1245/s10434-010-1431-3 